\title{
Multi-Scale Flight Path Planning for UAS Building Inspection
}

\author{
Paul Debus ${ }^{1 \text { [0000-0001-6228-588X] }}$, and Volker Rodehorst ${ }^{1}$ \\ ${ }^{1}$ Bauhaus-Universität Weimar, Germany \\ paul. debuscuni-weimar. de
}

\begin{abstract}
Unmanned aircraft systems (UAS) show large potential for the construction industry. Their use in condition assessment has increased significantly, due to technological and computational progress. UAS play a crucial role in developing a digital maintenance strategy for infrastructure, saving cost and effort, while increasing safety and reliability. Part of that strategy are automated visual UAS inspections of the building's condition. The resulting images can automatically be analyzed to identify and localize damages to the structure that have to be monitored. Further interest in parts of a structure can arise from events like accidents or collisions. Areas of low interest exist, where low resolution monitoring is sufficient.

From different requirements for resolution, different levels of detail can be derived. They require special image acquisition parameters that differ mainly in the distance between camera and structure. Areas with a higher level of detail require a smaller distance to the object, producing more images.

This work proposes a multi-scale flight path planning procedure, enabling higher resolution requirements for areas of special interest, while reducing the number of required images to a minimum. Careful selection of the camera positions maintains the complete coverage of the structure, while achieving the required resolution in all areas. The result is an efficient UAS inspection, reducing effort for the maintenance of infrastructure.
\end{abstract}

Keywords: UAS · Flight path planning $\cdot$ Infrastructure assessment $\cdot$ Imagebased SHM · Drones · Multi-scale monitoring

\section{Introduction}

The technological progress in the field of unmanned aircraft systems (UAS) in recent years has enabled many important applications of those mobile sensor platforms in all areas of life [1], especially for remote sensing [2]. UAS facilitate the fast and easy access to places that are not without problems reachable or dangerous for humans. Equipped with modern camera hardware, they allow for a fast and automated acquisition of image data, that can be used in many applications [3].

The maintenance of infrastructure is important for the functioning of a modern society. Many bridges and similar structures have been built in the last century and are nearing the end of their design life. In Germany, more than $60 \%$ of all road bridges 
are older than 30 years [4], while normal design life of a bridge is around 50 years. To allow for an efficient management of the necessary repair work, a digital maintenance strategy is required, enabling a high utilization of available resources. Part of this strategy has to be the support of human actors with digital technologies, modern data acquisition and processing tools and transparent and understandable decision aids.

The use of UAS for the condition assessment of large infrastructure has the potential to greatly reduce the effort, cost and time required for reliable monitoring [5], while increasing reliability and reproducibility of the results. The availability and mobility of UAS allow them to capture the surface of a structure far more efficiently and rapidly, than human inspectors could. In combination with modern data processing pipelines like Structure from Motion (SfM) [6] for three dimensional (3D) reconstruction or automated image analyses, they allow for a fast initial assessment of the condition of the structure. Especially modern machine learning tools enable the automated detection of anomalies like cracks and other damages to the structure [7]. Together with high-resolution deformations, computed from the 3D models, simulations and predictions of the current and future condition of the structure are possible and enable adequate maintenance [8].

One of the biggest advantages of this technology is to allow human bridge inspectors, the experts that are finally responsible for the correct maintenance, to focus their attention on relevant parts of the structure, after the UAS based inspection identified regions of high and low interest. Not only does this speed up the inspection process, it also allows to pay more attention to critical parts, where potential damages were detected in the images.

One challenge of the application of UAS-based visual inspection is the amount of data it generates. To cover the entire structure in high resolution, many images are required, that need to be processed for the analysis. To reduce the number of these images to a manageable amount, careful selection of the camera positions, from which the images are taken, is required. The task of UAS flight path planning has been part of active research in recent years, creating flight routes that cover the entire structure with the required resolution. The number of necessary images can still be very high, if a high resolution of the inspection is required.

One approach to aid with this is to not capture the entire structure with the same resolution, but to consider the relevance and interest of different parts of the structure for the flight path planning. If previous inspections detected areas where damages exist or are developing, those areas have to be monitored more closely than other parts where no damage is suspected. On the other hand, some areas may not need high resolution at all, for example parts that are not part of the supporting structure, like railings or noise barriers.

\subsection{State of the Art}

The application of UAS in the monitoring and inspection of infrastructure buildings has increased significantly in recent years [2] [3], especially in the field of visual inspection and remote sensing. In [9], the authors combine the application of UAS with 
bridge information modelling (BrIM) tools, to collect and aggregate valuable lifecycle information. The combination of UAS and installed monitoring systems is evaluated in [10], which shows that both technologies can successfully combined.

In a related publication [8], the authors of this work have proposed a framework for an integral digital maintenance strategy based on visual UAS inspections that also includes numerical simulations and virtual reality (VR) technologies, to increase the lifespan of bridges.

In a study for the State Department of Transportation in the United States [11], the authors compare different existing solutions with regard to their applicability and find that the principal applicability of UAS is high, while practical limitations hinder a wide adoption.

One important requirement for the application of UAS for inspection tasks is a suitable flight path. The full potential can only be used, if the mission is planned in advance to achieve all the goals of the inspection. Several approaches have been proposed, for example in [12], a bridge is partitioned into planar surfaces, for each of which a local route is computed that are later combined.

In [13], the authors introduce a method for flight planning that focuses on the image based 3D reconstruction, while also considering environmental limits, like no-fly zones. They show that their proposed method is able to compute flight paths that cover the structure of interest well for object resolutions of few centimeters.

The authors of [14] describe the application of UAS for the inspection of pipelines and canals, where the drone follows the long structures and conducts more detailed inspections of areas with detected anomalies. While this method works on a much larger scale, the findings support the approach proposed here, that different levels of inspection resolution can provide value, while saving time and effort.

\section{Multi-Scale Building Inspection}

One challenge of bridge inspection is the scale of the inspected structures combined with the necessity to inspect even small phenomena like cracks in concrete. One approach to handling this challenge is to not process the entire structure with the same detail, but to identify parts, that require higher resolution and inspect the rest with lower resolution. This reduces the overall effort for the inspection and allows focusing on parts that deserve attention.

This approach also values previous knowledge about a structure that would not be exploited, if the entire structure was inspected equally. If damaged areas have been detected in previous inspections, those require closer attention and need to be inspected with higher resolution. In addition, the expert knowledge and experience of human inspectors can be applied here. Inspectors often have a good knowledge, which parts of a structure are susceptible to damages and which parts generally do not suffer from damages visible in UAS images. Special parts of a structure, like bearings, hinges and joints suffer from strong wear over time and have to be inspected 
closely in most cases. On the other hand, non-bearing elements, like railings, do not require close inspection and can be covered with low-resolution inspection. This application of human expert knowledge can improve the automated inspection process.

In the case of an accident, for example the impact of a vehicle in a bridge pier, this specific area might need fast and precise inspection, if the stability of the structure is compromised. In this case, not only the directly impacted part is of interest, but also the entire structure, if deformations appear that are symptoms of deeper damage to the bridge.

\subsection{Definition of Levels of Interest}

We propose the definition of three interest levels, to not only mark regions of high interest that have to be inspected very closely, but also regions of explicitly low interest that can be covered with low resolution, as shown in Table 1. The object resolution is the size of one pixel in an image on the surface of the structure, equivalent to the smallest reliably measurable size in the resulting data. The object distance is computed from that target resolution under the assumption of the following properties of the used camera hardware:

- Model: Sony $\alpha$ 7R II

- Resolution: $7360 \mathrm{px} \times 4912 \mathrm{px}$

- Focal length: $50 \mathrm{~mm}$

While the defined levels of interest are very useful for this applications, they can be varied in other cases, according to the specific requirements of the inspection task. The resulting parameters are only inputs into the computation and can be changed for other values if required.

Table 1. Defined interest levels for the inspection and example purposes

\begin{tabular}{|l|c|r|l|}
\hline Interest Level & Object Resolution & Object Distance & Purpose \\
\hline 1 (low) & $2.0 \mathrm{~mm} / \mathrm{px}$ & $22.2 \mathrm{~m}$ & Rough geometry \\
\hline 2 (medium) & $1.0 \mathrm{~mm} / \mathrm{px}$ & $11.1 \mathrm{~m}$ & Detailed geometry \\
\hline 3 (high) & $0.1 \mathrm{~mm} / \mathrm{px}$ & $1.1 \mathrm{~m}$ & Crack detection \\
\hline
\end{tabular}

In most UAS-based inspection applications, the normal resolution level (Level 2) is realized, with $10 \mathrm{~m}$ being a distance to the object that common path planning procedures can generate [15]. An inspection of this level can be used to make measurements in the resulting 3D model, look for bigger damages that are well visible in the images and extract the 3D geometry of the structure for further analyses and simulations.

Another very common inspection level is the low interest inspection (Level 1), in many cases based on aerial images from above and some images from the sides, from a far distance away. These inspections allow for a visual overview for human inspectors that for example check if all required railings or traffic signs exist on a bridge. 
The low resolution only allows for rough measurements that are not sufficiently precise for simulation models.

The highest interest level (Level 3) is the most valuable for precise analysis of a buildings condition. It enables accurate image analysis for the detection and localization of damages, such as cracks or spallings, where even small shapes are well visible. The specific resolution of $0.1 \mathrm{~mm}$ was chosen based on the requirements of automated crack detection in the resulting images. For an object to be detectable in an image, it has to be contained in at least 2px according to the Nyquist-Shannon sampling theorem [16]. With one pixel corresponding to $0.1 \mathrm{~mm}$, a crack of $0.2 \mathrm{~mm}$ can reliably be discovered in images, creating a sensible trade-off between detecting all cracks and limiting the number of required images.

Each level on its own can be used for different inspection tasks, but the combination of those levels allows for a complete inspection of the entire structure, while providing high resolution for parts, where it is required.

\subsection{Derivation of Acquisition Parameters}

While the target resolution was defined in Section 2.1 for the different levels, the required distance between camera and object was computed using the properties of the used camera and the following equation

$\frac{d_{o b j}}{d i s t}=\frac{d_{p i x}}{f}$

Where $d_{o b j}$ is the smallest measurable distance on the object, dist is the distance between the camera and the object, $d_{p i x}$ is the size of one pixel on the sensor in the camera and $f$ is the focal length of the lens. With the assumption that one can measure with the precision of $1 \mathrm{px}$ in the image, the required distance between camera and object can be computed as

dist $=f \frac{d_{o b j}}{d_{p i x}}$

depending on the required object resolution $d_{o b j}$ in mm/px. For the camera used in this work with $d_{p i x}=0.00451 \mathrm{~mm} / p x$ and $f=50 \mathrm{~mm}$, this results in

dist $=d_{o b j} * 11086 p x$

for the computation of the values in Table 1.

One important result of the UAS-based structure inspection is the generation of 3D models. For this, the SfM pipeline, as shown in Fig. 1, is commonly used. This method exploits changing views of a scene while moving a camera, where the perspective distortion of objects in the images is different depending on their distance to 
the camera, to simultaneously compute the camera poses and the 3D geometry of the scene, from which the images where taken. It is based on finding corresponding points in multiple images, from which the relative orientation of the cameras to each other can be computed. By incremental combination of all image pairs, an image bundle can be constructed, which contains all camera positions and the identified object points in the same 3D space.



Fig. 1. The structure from motion pipeline [6]

For this process to work reliably and produce good results, the acquired images have to fulfill certain criteria. To find the same points in different images, those images have to overlap to a certain degree. In practice, an overlap of $70 \%$ in flight direction and $20 \%$ in the orthogonal direction have proven to be a good compromise between a stable reconstruction and a reduced number of images. Further, it is beneficial if points are visible in more than two images, to strengthen the image bundle and reduce errors.

\section{$3 \quad$ Flight Path Planning}

The goal of the flight path computation is to define an ordered set of camera positions and viewing directions, from which the structure that is to be inspected is completely covered with overlapping images in the defined resolution. Those viewpoints are then transferred as waypoints to the control of the UAS for the automated image acquisition.

The computation of the viewpoints is based on an existing 3D model of the structure. This model only needs to provide the approximate shape of the structure, precise to ca. $10 \mathrm{~cm}$, to be able to compute the paths with full coverage and no collisions. Those models can come from previous UAS inspections with low resolution, created using the same SfM pipelines as used for the high-resolution inspection. Another option is to use digital models created during the planning of the structure, if such models are available. Using for example a CAD, FEM or BIM model, the geometry of the structure can be extracted and transformed into a world coordinate system. This is required, as the computed camera positions have to be approached by the UAS using a GNSS, which operates in this coordinate system.

The proposed approach to computing the viewpoints is based on selecting only the minimal set of required camera positions from a larger set of possible camera positions. 


\subsection{Computation of Camera Positions}

The 3D model, on which the computation is based, is represented as a 3D polygon mesh of the structure. The mesh consists of a set of $n$ triangles $F$ called faces that form the surface of the structure. They are defined via a set of $m$ vertices $V$, that define the locations of the triangles, such that $V_{i ; 1}, V_{i ; 2}, V_{i ; 3}$ are the three vertices of face $F_{i}$.

To obtain a large set of possible camera positions that guarantee complete coverage of the model, a corresponding viewpoint $C_{i}$ is computed for each of the triangles $F_{i}$ of the mesh. Each face belongs to one of the levels of interest defined in Section 2.1 and therefore has an associated distance $f_{i}$, from which it has to be captured. For each face $F_{i}$, its center $c_{\mathrm{i}}$ and outward pointing unit normal vector $n_{i}$ can be computed via the average of the triangles vertices and the cross product of two its clockwise edge vectors as shown in the following equations.

$c_{i}=\frac{1}{3} \sum_{j=1}^{3} V_{i, j}$

$n_{i}=\left(V_{i, 1}-V_{i, 2}\right) \times\left(V_{i, 3}-V_{i, 2}\right)$

The position $C_{i}^{p}$ and the viewing direction $C_{i}^{d}$ corresponding to face $F_{i}$ are computed as follows

$$
\begin{gathered}
C_{i}^{p}=c_{i}+n_{i} * f_{i} \\
C_{i}^{d}=-n_{i} * f_{i}
\end{gathered}
$$

Each of the computed viewpoints is the defined distance away from the surface of the structure and its image contains a part of the structure. To compute the visible part of the surface, the vertices of the mesh are used as object points to represent the structure. The viewpoints are defined such that at least the three vertices of the corresponding face are visible, while generally more of the surrounding is visible in an image. To ensure the correct coverage of the surface, each vertex is only counted as visible in viewpoints corresponding to the highest interest level of its adjacent faces.

For the selection of the minimal subset of viewpoints that covers the entire structure, the complete visibility relations between the viewpoints and the object points has to be available. These relations are represented as a $m \times n$ visibility matrix $M$, with one column per viewpoint and one row per object point, where

$$
M_{i j}= \begin{cases}1 & \text { if point } \mathrm{i} \text { is visible from viewpoint } \mathrm{j} \\ 0 & \text { otherwise }\end{cases}
$$


This matrix is computed column wise, for each viewpoint individually, in three steps:

1. Selection of only those object points, whose interest level is equal the interest level of the considered viewpoint.

2. Projection of all remaining object points into the image, to select only those points, whose projections lie in the image.

3. Computation of intersections of the corresponding viewing rays with the structure. If the first intersection is closer than the original point, something is occluding the point from the camera and the point is discarded. If the first intersection is the original point, the point is visible in the image and the corresponding entry in the $M$ matrix is set to 1 .

$S_{i}$ is the set of object points, that are visible from viewpoint $C_{i}$ and is equivalent to the 1 entries in $M_{: i}$, the $i$-th column of $M$.

\subsection{Selection of the Required Camera Positions}

With the computed visibility matrix $M$, the selection of the required camera positions can be described as a set multi-cover problem as stated in [17]: "Given a universe $V$ of $m$ vertices and a collection of sets $S=\left\{S_{1}, \ldots, S_{n}\right\}$, where each $S_{i}$ is a subset of $V$, find a minimum cardinality sub-collection $R \subseteq S$, such that $R$ covers $V$; in other words, the union of the sets in $R$ is $V$. [...] In [the set multi-cover] version, each element $e \in V$ has an integer demand or requirement $d(e)$ and a multi-cover is a sub-collection $R \subseteq S$ such that for each $e \in V$ there are $d(e)$ distinct sets in $\mathrm{R}$ that contain $e . " 1$

The demand $d(e)$ defines the number of images, each object point has to be visible in. This value can be derived from the requirements of the photogrammetrical 3D reconstruction using SfM. As described in Section 2.2, a point has to be visible in at least two images for the 3D reconstruction to work. The process can be improved and the result stabilized by finding correspondences in more than two images, which also allows for deviations in the image acquisition and other inaccuracies in the process. For these reasons, a constant demand of $d(e) \dot{i} \forall e \in V$ is defined for all points.

The set multi-cover problem is NP-HARD [17], no polynomial time exact algorithms are available, so it is generally solved using approximation algorithms. This topic has been part of active research in recent years, and many approaches have been proposed [17] [18] [19] [20]. In this work, a simple greedy algorithm, shown in Algorithm 1, is used that takes the visibility matrix $M$ as input and produces a set of viewpoints that cover the entire structure.

1 Symbols changed to match those used in this work 
Algorithm 1. Greedy set multi-cover algorithm

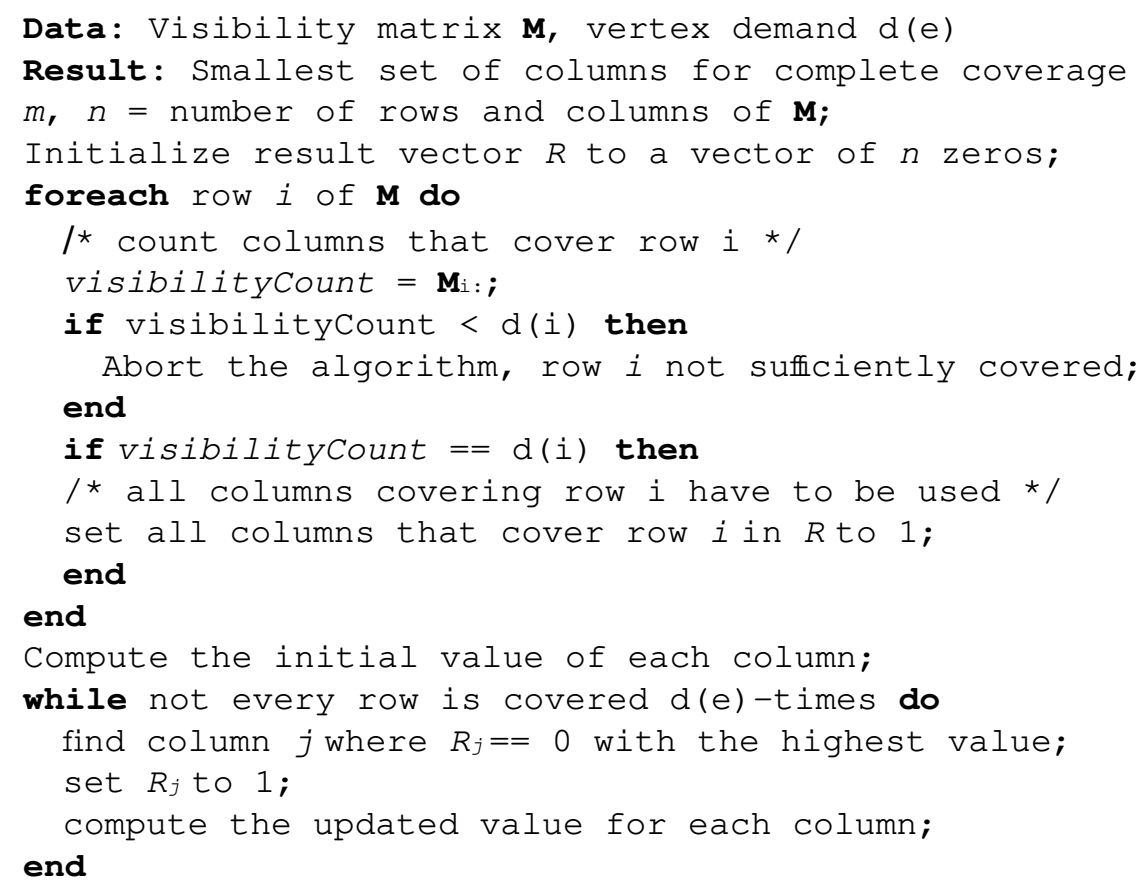

The algorithm starts with fixing those viewpoints, that need to be included, because some of the object points they contain are only visible in $d(e)$ images. Then the next viewpoint with the highest value is chosen iteratively, until complete coverage is reached. The value of a column is the number of rows it covers, that have not been sufficiently covered by the already selected columns.

After computing the set of viewpoints, a flight path connecting them has to be computed. This is equivalent to the order, in which the UAS will approach the viewpoints during the automated image acquisition. The computation has to not only find an efficient connection between all positions, but also consider some practical aspects. The route may not come close to, or even touch, objects, either of the structure or the surrounding. These collisions have to be avoided under all circumstances. Further, the flight duration of the UAS is limited, forcing multiple sub-routes instead of one continuous route. With these considerations, the path-planning problem can be modeled as a distance constrained vehicle routing problem, a special case of the traveling salesperson problem. This sort of problems is well described in literature and many solution approaches exist. In this case, the route planning is solved using the LinKernighan-Helsgaun solver [21]. 


\section{Application of the Method}

The proposed method aims at being a general approach, to computing flight paths for visual UAS inspections of structures. Especially since no implicit assumptions about the structure, the geometry or the mesh used for the computation are made, the method can be applied to a variety of structures. For practical validation of the approach, a reference object has been selected, for which flight routes have been computed that include defined regions of interest based on real observations. The results have been evaluated to show the applicability of the proposed method.

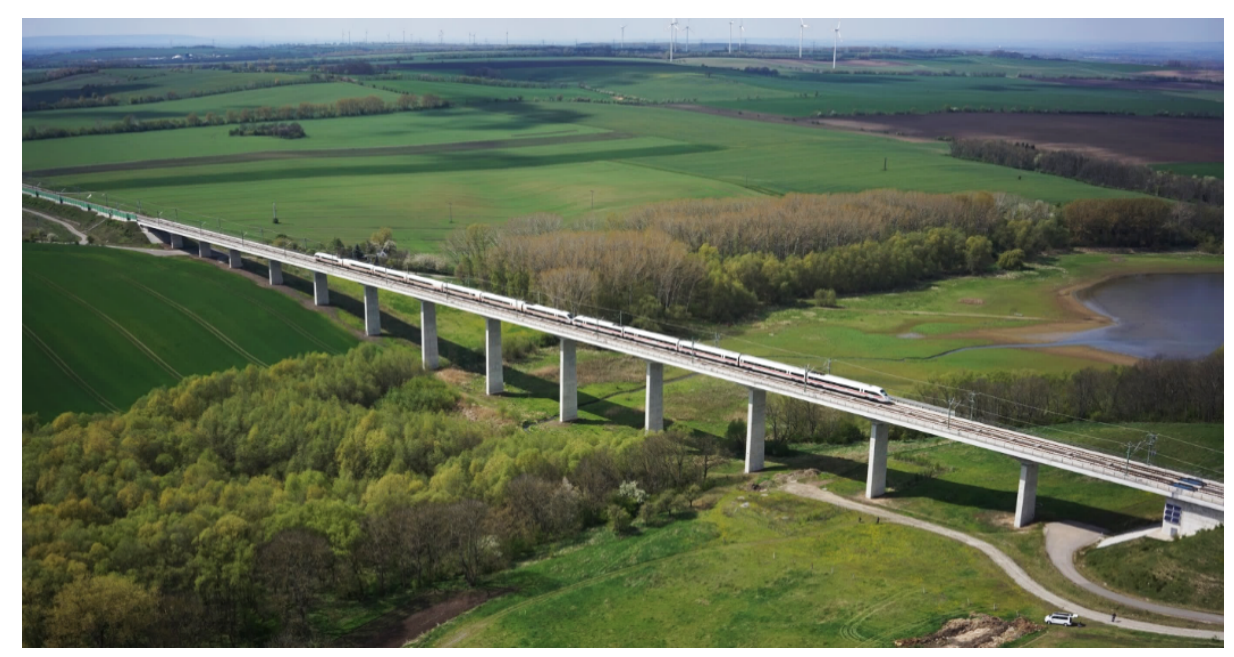

Fig. 1. The Scherkonde bridge, reference for the validation of the method

\subsection{The Reference Object}

For the validation of the proposed method, the Scherkonde bridge shown in Fig. 1 that is located on the railway high-speed track between Erfurt and Berlin, Germany, has been selected. The bridge was completed in 2011 after three years of construction and is built in semi-integral pre-stressed concrete with monolithic conjunctions between the superstructure and the supports. This leads to large horizontal forces in the piers, which require close monitoring. The bridge has a length of $576 \mathrm{~m}$ and 13 piers, the highest of which measures $34 \mathrm{~m}$. 




Fig. 1. Image acquired during a previous UAS inspection


Fig. 1. Detail section and hypothesis of possible cracks on the surface

The bridge has been object to previous UAS inspection missions, so image data, simulations and 3D models are available. Fig. 1 shows an image from one of those inspections in 2019, containing the center part of a pier. Fig. 1 shows a section of that image in full resolution, approximately $1 \mathrm{~mm} / \mathrm{px}$ on the surface. The vertical planking patterns have a width of around $10 \mathrm{~cm}$ or 100px. Using the method described in [22], a preliminary crack detection on the images using Convolutional Neural Networks (CNN) has been performed. The CNN produced a response for two regions of the image, where cracks could be located. Similar effects occur in other regions of the image. Due to the low resolution of the image, no precise detection is possible, so the result is vague and could well be a false positive detection. 




Fig. 1. Reconstructed 3D model of a part of the Scherkonde bridge, created from previous UAS inspections

In addition to the analysis of the images, a 3D model has been computed using the SfM pipeline. A section of that model is shown in Fig. 1.That part consists of 500000 triangles and 250500 vertices, contains the pier that is shown in Fig. 1 and is used in the following as the reference model.

\subsection{Assignment of Interest}

Even though the bridge under consideration is relatively new, it is subject to reoccurring inspections as a maintenance measure. In a previous UAS inspection, images of the bridge have been captured and analyzed. In images of one pier, the automated crack detection showed signs of possible damages to the structure. To generate a new data set, that provides higher resolution coverage of the possibly damaged area, an adaptive multi-scale flight path is to be computed.

The multiple scales for the flight path are defined equivalent to the levels defined in Section 2.1, as they each provide the required amount of information and resolution for the different parts of the structure. The area of the pier, that is visible in Fig. 1, shows a hypothesis for possible cracks on the surface and has to be monitored closely, so it is assigned to the highest level of interest (Level 3). The rest of that pier could also be in a condition that requires more attention, so it is assigned the medium level of interest (Level 2), while the rest of the model is assigned to Level 1, as it is only required for completeness and context. This context is especially important for the geo- 
registration of the $3 \mathrm{D}$ model computed from the images, as the points with precisely measured locations are distributed over the entire structure and are generally not all contained in images of the higher interest levels.

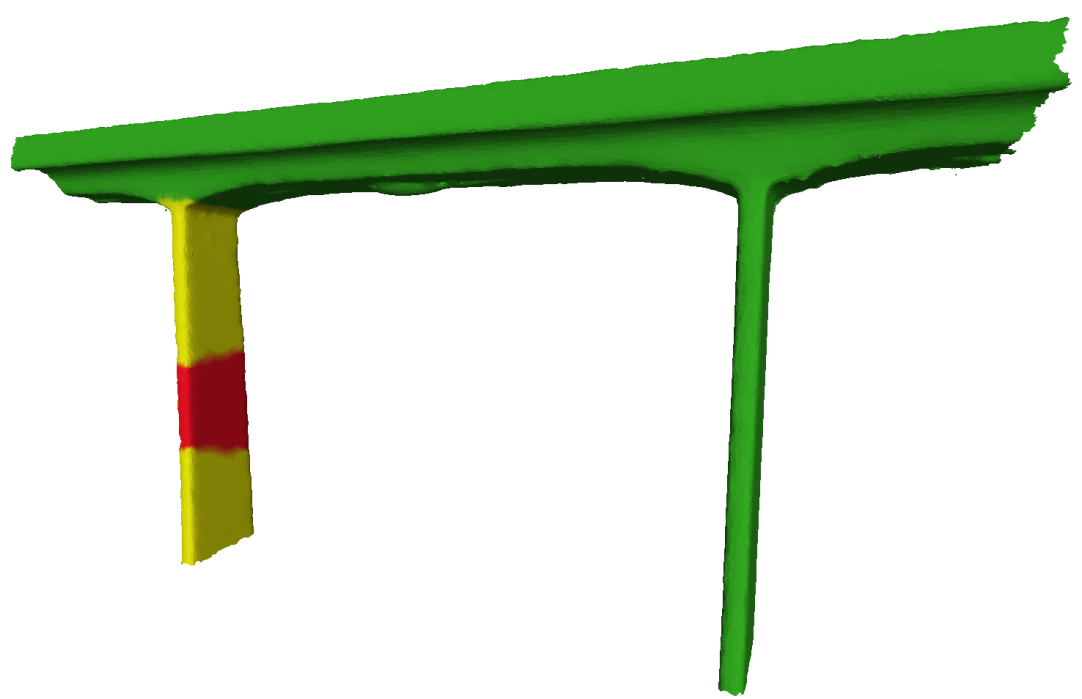

Fig. 1. Model of the Scherkonde bridge with faces colored according the level of interest

Fig. 1 shows the model of the bridge, colored according to the defined interest levels. As the 3D model was computed using SfM, the orientation of the images is also known. With this knowledge, the exact visible part of the pier from the image of the damaged area can be computed and is highlighted in red.

\subsection{Resulting Flight Path}

After assigning the interest levels to the different parts of the structure, the flight path was computed according to the method described in Section 6. Table 1 shows the resulting number of viewpoints for each interest level, together with the corresponding area that needs to be covered.

Table 1. Interest levels and corresponding viewpoints

\begin{tabular}{|l|r|r|l|}
\hline Level & Area & Viewpoints & Density \\
\hline 1 & $2796 \mathrm{~m}^{2}$ & 122 & $0.04 \mathrm{vp} / \mathrm{m}^{2}$ \\
\hline 2 & $372 \mathrm{~m}^{2}$ & 62 & $0.17 \mathrm{vp} / \mathrm{m}^{2}$ \\
\hline 3 & $58 \mathrm{~m}^{2}$ & 281 & $4.84 \mathrm{vp} / \mathrm{m}^{2}$ \\
\hline Total & $3225 \mathrm{~m}^{2}$ & 465 & $0.14 \mathrm{vp} / \mathrm{m}^{2}$ \\
\hline
\end{tabular}


The table shows that reducing the interest level for large parts of the structure, which are indeed of lower interest, greatly reduces the number of required images. High interest inspections produce around 120 times more viewpoints for the same area, than low interest inspections $\left(0.04 \mathrm{vp} / \mathrm{m}^{2} \mathrm{vs} .4 .84 \mathrm{vp} / \mathrm{m}^{2}\right)$. This corresponds well with the fact that the area visible in an image grows with the square of the distance to the surface. Had the entire structure been captured in high resolution, more than 15000 images would have been required, compared to the 465 that are the result of the adaptive multi-scale flight path.

\subsection{Validation of the Damage Hypothesis}

After computing a flight path for the detailed and targeted inspection of the possibly damaged area, an automated UAS inspection was performed on the bridge. Using the computed waypoints and the GNSS navigation system, the second UAS inspection was conducted shortly after the initial mission.

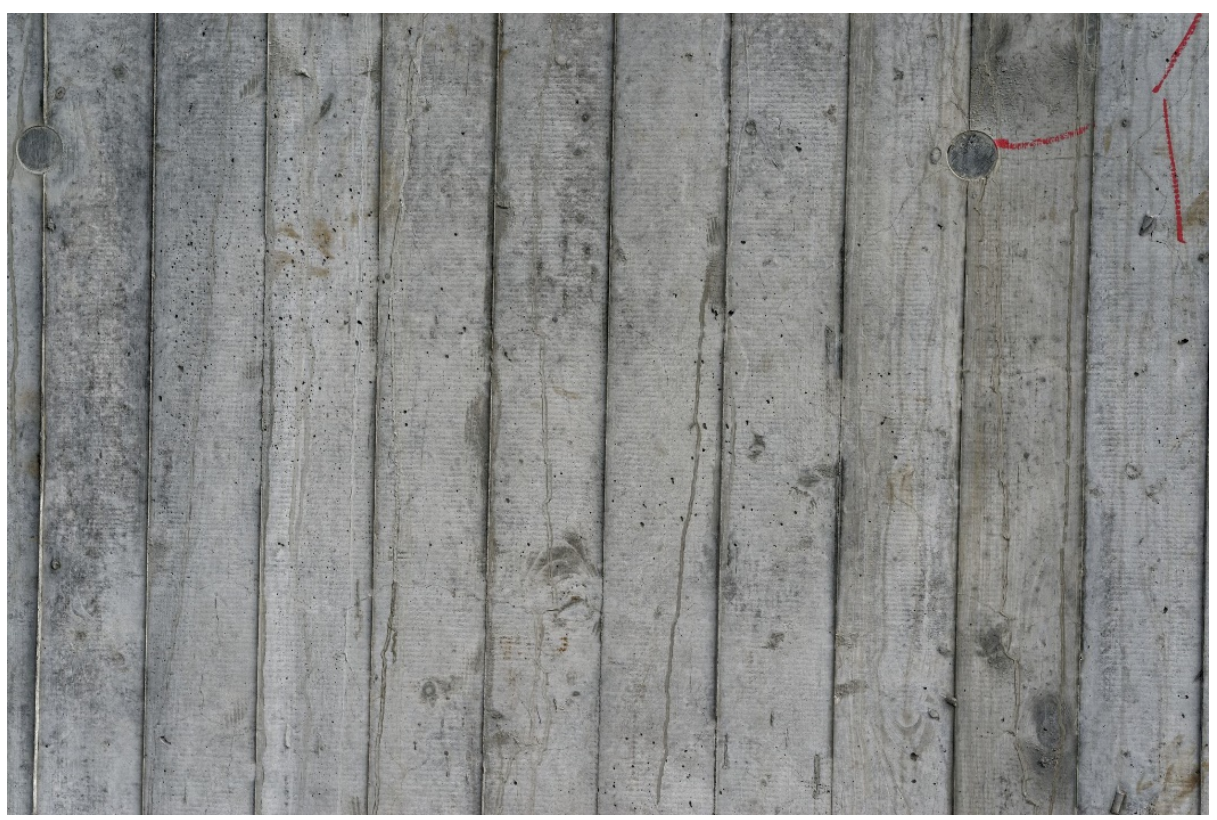

Fig. 1. Image from the resulting high-resolution flight path acquired from ca. 1m 


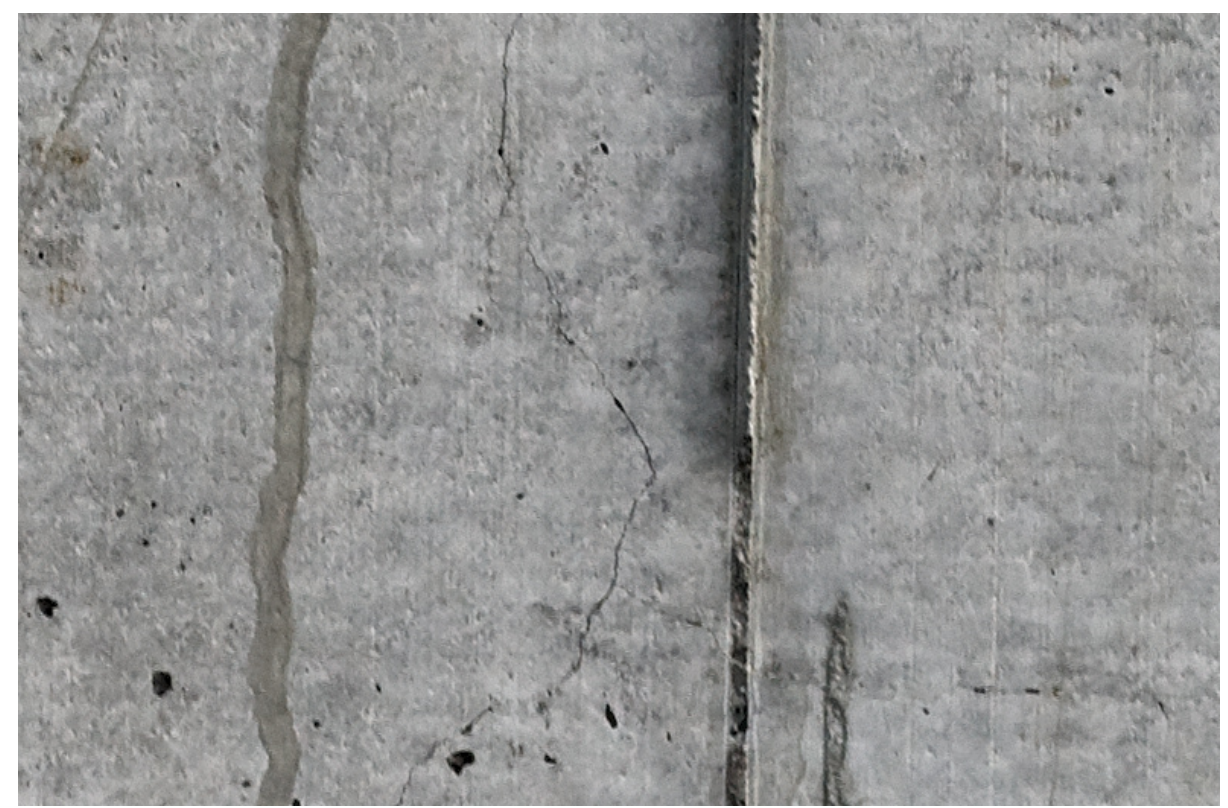

Fig. 1. Detail of the high-resolution image in the original size containing a small crack

The evaluation of the newly created images allowed a closer inspection of the surface. Fig. 1 shows one of the resulting images with a full resolution detail of the area, where a possible damage had been suspected previously, shown in Fig. 1. Running the automated damage detection on those images showed that one of the detections was a false positive that was caused by a mud stain on the surface. The other hypothesis, which is visible in Fig. 1, was correct and corresponded to a crack in the concrete. With the high resolution however, it was determined by an engineer that the crack is not relevant for the safety and structural health of the bridge, the width corresponding to roughly $0.15 \mathrm{~mm}$. It is only a small damage to the surface that does not have any further implications for the structure. This shows the possibilities this new technology provides, enabling the human experts to quickly obtain high-resolution information about regions of interest. In this case, the suspected damages were either false alarms or not relevant, but in other cases a disaster could be prevented by having an early warning system for damages, before they happen. 


\section{Conclusion}

In this paper, a method for the computation of flight paths for visual UAS building inspection is proposed that takes into account different levels of interest defined for parts of a structure. In Section 2.1, the levels of interest are defined as high interest for areas that need very close attention, medium interest for areas that have to be well visible in the resulting images, and low interest for areas that are not important for the inspection itself, but for context information and completeness, especially for the georeferencing of the reconstructed 3D model. The assignment of a certain interest to parts of the structure can be based on many factors, like expert knowledge of the structure or an impact event. One important reason to mark an area for high interest can be the suspicion of cracks or other damages that can be detected in images.

For each of the defined levels of interest, viewpoints have to be computed that cover the corresponding area well, while reducing the number of images to a minimum. For this, a method is proposed that is based on selecting a minimum covering set from a larger set of possible camera positions. This large set is computed by assigning one viewpoint to each triangle of the model of the structure. Using a simple greedy solver for the set multi-cover problem, good solutions can be computed quickly.

A case study has been conducted, in which information from a previous inspection was used to compute a flight path that provides special attention to areas that could be damaged. Automated image analysis of the low detail images indicated the possibility of cracks in the concrete surface. After conducting the high resolution and detailed inspection of the identified area, it showed that the suspected damages were either false alarms or small surface cracks without any implications for the structural health and safety. The method itself proved to work well for the generation of flight paths that cover specific areas of a structure in high resolution for details, while also covering the other parts of the structure in lower resolution for context.

This shows that visual UAS monitoring can help protect important infrastructure by quickly inspecting large structures, allowing for automated analysis of the results and the dispatch of more detailed inspections, should possible damages be detected. As part of a digital maintenance strategy, it helps human experts to focus on the important aspects of the structures, while making sure that nothing is missed.

\section{Further research possibilities}

While the presented approach already produces good results for the studied test cases, the wide variety of building shapes can introduce further challenges that need to be overcome. The current implementation does not try to incorporate the surrounding of the structure while placing the possible viewpoints, but only during the computation of the visibility relations. Changing this would allow placing closer viewpoints in regions where space is limited and the computed larger distance between camera and object is not possible. 
Currently, a very simple greedy solver for the set multi-cover problem is used. A more advanced solver could provide solutions that are closer to the mathematical optimum. The solution quality can also be improved by incorporating special knowledge concerning UAS flights and photogrammetric reconstructions as well as automated image analysis. While this may not influence the mathematical quality of the solutions, the real world applicability can greatly benefit from adapting the solution algorithm to additional constraints.

At the moment, the selection of a viewpoint does not affect the value of surrounding viewpoints, while in an improved version, viewpoints very close to already selected points should be discouraged, to produce a sufficiently large baseline (distance) between adjacent viewpoints. Further attention should be paid to special geometric features of the structure, for example corners or very fine details. For a stable SfM reconstruction, adjacent images may not be too different from each other, especially with regard to rotation, as otherwise the matching of corresponding feature points becomes very difficult.

While the 3D reconstruction could be computed successfully in the studied cases, the reconstruction at the borders between the interest levels showed some artifacts, especially between levels 2 and 3, where the distances increases by factor 10 . In these areas, additional viewpoints with intermediate distances could help improving the quality of the reconstruction, while increasing the number of images only slightly.

\section{$7 \quad$ Acknowledgement and Funding}

This work was supported by the German Federal Ministry of Education and Research as part of the AISTEC project under grant numbers 13N14657 to 13N14660. We would also like to thank our project partners, especially the Deutsche Bahn, for making this work possible. 


\section{References}

[1] H. Shakhatreh, A. H. Sawalmeh, A. Al-Fuqaha, Z. Dou, E. Almaita, I. Khalil, N. S. Othman, A. Khreishah and M. Guizani, "Unmanned Aerial Vehicles (UAVs): A Survey on Civil Applications and Key Research Challenges," IEEE Access, vol. 7, pp. 48572-48634, 2019.

[2] T.-Z. Xiang, G.-S. Xia and L. Zhang, "Mini-Unmanned Aerial VehicleBased Remote Sensing: Techniques, applications, and prospects," IEEE Geoscience and Remote Sensing Magazine, vol. 7, pp. 29-63, Sep 2019.

[3] F. Nex and F. Remondino, "Preface: Latest Developments, Methodologies, and Applications Based on UAV Platforms," Drones, vol. 3, p. 26, \#mar\# 2019.

[4] Bundesanstalt für Straßenwesen, "Zustandsnoten der Brücken," 2019. [Online]. Available: https://www.bast.de/BASt_2017/DE/Statistik/Bruecken/Zustandsnoten-excel.html.

[5] D. Reagan, A. Sabato and C. Niezrecki, "Feasibility of using digital image correlation for unmanned aerial vehicle structural health monitoring of bridges," Structural Health Monitoring, vol. 17, pp. 1056-1072, Oct 2017.

[6] J. L. Schonberger and J.-M. Frahm, "Structure-from-Motion revisited," in Proceedings of the IEEE Conference on Computer Vision and Pattern Recognition, 2016.

[7] A. Mohan and S. Poobal, "Crack detection using image processing: A critical review and analysis," Alexandria Engineering Journal, vol. 57, pp. 787798, Jun 2018.

[8] G. Morgenthal, N. Hallermann, J. Kersten, J. Taraben, P. Debus, M. Helmrich and V. Rodehorst, "Framework for automated UAS-based structural condition assessment of bridges," Automation in Construction, vol. 97, pp. 77-95, Jan 2019.

[9] V. Barrile, G. Candela, A. Fotia and E. Bernardo, "UAV Survey of Bridges and Viaduct: Workflow and Application," in Computational Science and Its Applications - ICCSA 2019, vol. 11622, Cham, Springer International Publishing, 2019, p. 269-284.

[10] D. Agdas, J. A. Rice, J. R. Martinez and I. R. Lasa, "Comparison of Visual Inspection and Structural-Health Monitoring As Bridge Condition Assessment Methods," Journal of Performance of Constructed Facilities, vol. 30, no. 3, 2016.

[11] S. Dorafshan, M. Maguire, N. V. Hoffer and C. Coopmans, "Challenges in bridge inspection using small unmanned aerial systems: Results and lessons learned," IEEE, p. 1722-1730. 
[12] K. Yu, P. Shanthakumar, J. Orevillo, E. Bianchi, M. Hebdon and P. Tokekar, View Planning and Navigation Algorithms for Autonomous Bridge Inspection with UAVs, p. 9.

[13] T. Koch, M. Körner and F. Fraundorfer, "Automatic and SemanticallyAware 3D UAV Flight Planning for Image-Based 3D Reconstruction," Remote Sensing, vol. 11, no. 13, p. 1550, 2019.

[14] R. Martin, L. Blackburn, J. Pulsipher, K. Franke and J. Hedengren, "Potential Benefits of Combining Anomaly Detection with View Planning for UAV Infrastructure Modeling," Remote Sensing, vol. 9, no. 5, p. 434, 2017.

[15] C. Peng and V. Isler, "Adaptive View Planning for Aerial 3D Reconstruction," in 2019 International Conference on Robotics and Automation (ICRA), 2019.

[16] C. E. Shannon, "Communication in the Presence of Noise," Proceedings of the IRE, vol. 37, pp. 10-21, jan 1949.

[17] C. Chekuri, K. L. Clarkson and S. Har-Peled, "On the set multi-cover problem in geometric settings," in Proceedings of the 25th annual symposium on Computational geometry - SCG 09, 2009.

[18] C. Chekuri and K. Quanrud, "On approximating (sparse) covering integer programs," in Proceedings of the Thirtieth Annual ACM-SIAM Symposium on Discrete Algorithms, 2019.

[19] A. Chen, D. G. Harris and A. Srinivasan, "Partial resampling to approximate covering integer programs," in Proceedings of the twenty-seventh annual ACM-SIAM symposium on Discrete algorithms, 2016.

[20] I. Dinur, V. Guruswami, S. Khot and O. Regev, "A new multilayered PCP and the hardness of hypergraph vertex cover," SIAM Journal on Computing, vol. 34, pp. 1129-1146, 2005.

[21] K. Helsgaun, "An extension of the Lin-Kernighan-Helsgaun TSP solver for constrained traveling salesman and vehicle routing problems," Roskilde: Roskilde University, 2017.

[22] C. Benz, P. Debus, H. K. Ha and V. Rodehorst, "Crack Segmentation on UAS-based Imagery using Transfer Learning," in 2019 International Conference on Image and Vision Computing New Zealand (IVCNZ), 2019. 